\title{
Commensurability Effects in Large Josephson Junctions
}

\author{
Leon Balents \\ Institute for Theoretical Physics, University of California, Santa Barbara, CA 93106-4030 \\ Steven H. Simon \\ Department of Physics, Harvard University, Cambridge, MA 02138
}

\begin{abstract}
Two types of commensurability effects are possible in a large Josephson junction patterned with columnar defects. The first occurs for a periodic array of pins when the mean fluxon spacing (tuned by the magnitude of the applied in-plane magnetic field) is a rational fraction of the defect spacing. We show that this effect leads, under fairly general conditions, to a mapping of the behavior of the Josephson junction near the commensurate field values to that of a zero field junction with an effective Josephson penetration depth. The second occurs for more general arrangements of pinning sites, when the orientation of the Josephson vortex lattice (tuned by the direction of the applied field) nearly matches the orientation of the defects. We investigate this tilt response in the limit of a single Josephson vortex. The results are compared, where possible, to recent experiments. As an aside from our main analysis, we prove that, contrary to recent claims in the literature, the critical current density vanishes in the thermodynamic limit, even in the presence of (non-pathologically distributed) pinning disorder.
\end{abstract}

\section{INTRODUCTION}

The pursuit of large critical current densities in high temperature superconductors in external magnetic fields has stimulated many investigations into flux pinning. Such pinning, caused by naturally occurring and/or artificially induced non-superconducting defects, is necessary to prevent dissipative flux flow in response to an applied current. At low temperatures in three dimensions, disorder is expected to induce glassy phases, in which the current-voltage relation is strongly non-linear, with vanishing linear resistivity. These states for point and line pins have been named the vortex glass 1 and Bose glass, ${ }^{1}$ respectively. Experimentally, continuous normalglass trafsitions have been observed for both types of disorder. 3 ⿶

Quantitative comparison with the theory is, however, difficult for several reasons. On the theoretical side, neither the critical exponents nor the properties of the low temperature phase are reliably known. 5 Experimentally, it is hard to prepare large samples with well characterized disorder. It is probably this difficulty which has lead to a fairly broad range of measured values for these critical exponents.

Josephson junctions (JJs) offer a promising alternative. By orienting a magnetic field parallel to the junction interface, the experimenter can create Josephson vortices in the junction, which then acts in many ways like a $1+1$ dimensional version of the bulk vortex state. Because of the sophistication and ease of modern fabrication techniques, samples may be engineered with almost any configuration of defects in an extremely controlled way. The theoretical situation is also much more secure for $1+1$ dimensional glasses, mainly due to the ability to perform renormalization group calculations.6 Large scale numeri- cal simulation in $1+1$ dimensions are also more tractable than their $2+1$ dimensional counterparts.

Patterned JJs are interesting in theirown right, and have been studied by several authors 19 Here, we discuss the case of linear (columnar) defects, focusing on commensurability effects tuned by changes in the magnetic field. Because of the relative ease of controlling the magnetic field versus other system parameters (e.g. temperature, disorder), such effects provide a convenient probe of the properties of the JJ.

The remainder of the paper is organized as follows. In section III, we review the properties of large uniform JJs and the equations describing their behavior. Section IIII describes how defects are incorporated into this model. In addition, we prove that, other than for particularly pathological cases, the introduction of defects does not allow a non-zero average critical current density for the JJ in the thermodynamic limit, contrary to recent claims in the literature 8 . Commensurability effects which occur when varying the magnitude of the applied magnetic field in a periodic defect array are explained in section IV. Extensive experiments on such systems have been carried out in Refs. 10 and 11, in good agreement with the theoretical results presented here. A discussion of complex commensurate states which occur at 1/2-integral fields is included in Appendix A.

A qualitatively different type of commensurability effect occurs upon changing the direction of the magnetic field. In section V, we calculate this tilt response for an isolated flux line, where the well-known analogy 12 to a one dimensional system is particularly useful. In section VI, we conclude with a discussion of the experimental checks on this work and its ramifications for future research. 


\section{LARGE JOSEPHSON JUNCTIONS}

A Josephson junction is an insulating interface between two (usually identical) superconductors. For simplicity, we will restrict the discussion to the so-called overlap geometry, shown in Fig.1. This is a common experimental choice, but by no means unique. Other configurations behave similarly, but require different boundary conditions.

In a JJ, all the quantities of interest may be expressed in terms of the gauge-invariant phase difference $\gamma \equiv \theta_{1}-\theta_{2}-2 \pi / \phi_{0} \int_{-d / 2}^{d / 2} d z A_{z}(z)$ between the two superconductors, where $\phi_{0}=h c / 2 e$ is the flux quantum. 13 Here $\theta_{1}$ and $\theta_{2}$ are the phases of the BCS order parameters in the top and bottom superconducting slab, $A_{z}$ is the $\mathrm{z}$-component of the vector potential. The length $d$ is the magnetic thickness of the junction, and is related to the actual thickness $s$ by $d=s+2 \lambda$, where $\lambda$ is the London penetration depth. The current density in the $z$-direction has the Josephson form

$$
j_{z}=j_{0} \sin \gamma .
$$

We will regard the tunneling current density $j_{0}$ as an experimental constant. The magnetic field components parallel to the junction plane are

$$
\begin{aligned}
& B_{x}=-\frac{\phi_{0}}{2 \pi d} \frac{\partial \gamma}{\partial y} \\
& B_{y}=\frac{\phi_{0}}{2 \pi d} \frac{\partial \gamma}{\partial x}
\end{aligned}
$$

Assuming a static situation in the interior of the junction, Eqs.2.1 and 2.2 can be combined with the Maxwell equation $\nabla \times \mathbf{B}=4 \pi \mathbf{j} / c$ to yield

$$
\lambda_{J}^{2} \nabla^{2} \gamma=\sin \gamma,
$$

where $\nabla^{2}$ indicates the two dimensional Laplacian in the interface plane. The length $\lambda_{J}=\sqrt{c \phi_{0} / 8 \pi^{2} d j_{0}}$ is known as the Josephson penetration depth. Eq.2.3 is known as the (time independent) sine-Gordon equation.

Assuming the current is injected parallel to the $y$-axis, the boundary conditions augmenting Eq.2.3 are 14

$$
\begin{aligned}
\left.\frac{\partial \gamma}{\partial x}\right|_{x=0} & =\frac{2 \pi d H_{y}}{\phi_{0}} \\
\left.\frac{\partial \gamma}{\partial x}\right|_{x=W} & =\frac{2 \pi d H_{y}}{\phi_{0}} \\
\left.\frac{\partial \gamma}{\partial y}\right|_{y=0} & =-\frac{2 \pi d H_{x}}{\phi_{0}}-\frac{4 \pi^{2} d I}{c \phi_{0} W} \\
\left.\frac{\partial \gamma}{\partial y}\right|_{y=L} & =-\frac{2 \pi d H_{x}}{\phi_{0}}+\frac{4 \pi^{2} d I}{c \phi_{0} W}
\end{aligned}
$$

Eq.2.3, in conjunction with Eq.2.4, can have multiple solutions. In such cases, the equilibrium situation is found by minimizing the free energy

$$
F=\epsilon_{\mathrm{J}} \int d x d y\left\{\frac{1}{2}|\nabla \gamma-\mathbf{h}|^{2}+\lambda_{J}^{-2}(1-\cos \gamma)\right\},
$$

where $\epsilon_{J}=\phi_{0}^{2} / 16 \pi^{3} d$ is the overall energy scale and $\mathbf{h}=$ $2 \pi d\left(-H_{y}, H_{x}\right) / \phi_{0}$.

We next briefly summarize the properties of a defect free junction. 15 In the absence of an applied magnetic field, a small or long junction with $L \lesssim \lambda_{J}$ can carry a current $I=L W j_{0} \sin \gamma$, with $\gamma$ approximately uniform, leading to a critical current $I_{c 0}=L W j_{0}$. This can be seen by transforming away the boundary conditions according to $\gamma \rightarrow \gamma+I(y-L / 2)^{2} /\left(2 j_{0} L W \lambda_{J}^{2}\right)$. For large junctions, where both $W, L \gg \lambda_{J}$, the uniform approximation fails. In this limit, the phase (and therefore the field and current) decays exponentially to zero in the interior of the junction. This screening is a property of the linearized sine-Gordon equation,

$$
\lambda_{J}^{2} \nabla^{2} \gamma \approx \gamma
$$

In this regime, $I_{c} \propto \lambda_{J}^{2} j_{0}$.

In an applied magnetic field, the critical current is further reduced. For strong fields, $H \gg \phi_{0} / d \lambda_{J}$, screening is negligible, and the phase winds approximately linearly transverse to the field axis, e.g.

$$
\gamma \approx \hat{\gamma}+\frac{2 \pi d}{\phi_{0}} H_{y} x, \text { for } H_{x}=0, H_{y} \gg \phi_{0} / d \lambda_{J} .
$$

In this limit, the critical current, obtained most simply by integrating Eq2.1, takes the Fraunhofer form

$$
I_{c} \approx j_{0} L W\left|\frac{\sin \left(\pi d H_{y} W / \phi_{0}\right)}{\pi d H_{y} W / \phi_{0}}\right| .
$$

For $H \gtrsim \phi_{0} / d \lambda_{J}$, the field penetrates the junction, but not uniformly. Instead, it gathers into "Josephson vortices" or "solitons" of total flux $\phi_{0}$, across which $\gamma$ changes by $2 \pi$. For large fields, these vortices comprise only a weak sinusoidal modulation of the field in the junction (as can be obtained by perturbation theory from Eq.2.3).

As $H \rightarrow H_{c 1 J}$, with $H_{c 1 J}=2 \phi_{0} / \pi^{2} d \lambda_{J}$, however, the solitons sharpen into objects of definite width $\lambda_{J}$, between which $\gamma$ rapidly decays to a multiple of $2 \pi$. For $\left(H-H_{c 1 J}\right) / H_{c 1 J} \ll 1$, a kind of critical phenomenon, known as a commensurate-incommensurate transition (CIT), governs the vanishing density of Josephson vortices. In this regime, it is legitimate to treat the solitons as weakly interacting, since their average separation is much larger than $\lambda_{J}$. The scaling of the free energy of an isolated Josephson vortex can be read off from Eq.2.5. By using the exact one-soliton solution to Eq.2.3,

$$
\gamma_{0}(x)=4 \tan ^{-1}\left(\exp \left(x / \lambda_{J}\right)\right)
$$

and subtracting the free energy for a configuration with no vortex, the full expression including the prefactor is found as 


$$
F_{\text {vortex }}=-8\left(H / H_{c 1 J}-1\right) \frac{L}{\lambda_{J}} \epsilon_{J} .
$$

For $H>H_{c 1 J}$, of course, inter-soliton interactions must be included to prevent the vortices from proliferating. In addition, we will also allow for small fluctuations in the positions and shapes of the vortices, since sufficiently small deformations of $\gamma$ cost a free energy less than $k_{\mathrm{B}} T$. Doing so leads to (neglecting an unimportant constant term) the soliton free energy (for $N$ vortices)

$$
\begin{aligned}
\mathcal{F}_{N}= & -N\left(H / H_{c 1 J}-1\right) \tilde{\epsilon} L+\int_{0}^{L} d y\left\{\sum_{n} \frac{\tilde{\epsilon}}{2}\left|\frac{d x_{n}(y)}{d y}\right|^{2}\right. \\
& \left.+\tilde{\epsilon} \sum_{n, n^{\prime}} V\left(\left|x_{n}(y)-x_{n^{\prime}}(y)\right| / \lambda_{J}\right)\right\},
\end{aligned}
$$

where $\tilde{\epsilon} \equiv 8 \epsilon_{J} / \lambda_{J}$, and $V(\chi) \sim 2 \chi \exp (-\chi)$ is an exponentially decaying repulsive interaction with magnitude and range of order unity (we have approximated the interactions as local in $y$ which is adequate for the dilute limit considered here). 10

To understand the vanishing of the vortex density at the CIT, Eq.2.11 is used to estimate the free energy as a function of $\ell$, the average fluxon separation. At finite temperature, an entropic contribution must be included due to the wandering of the solitons along the $y$ axis. 17 By equipartition, an individual Josephson vortex wanders according to $\left\langle(x(y)-x(0))^{2}\right\rangle \sim k_{\mathrm{B}} T y / \tilde{\epsilon}$. Each time the fluxon wanders a transverse distance $\ell$, it is caged by another soliton, and must reverse direction Naively, this constraint excludes half of the available configurations of the vortex. Taking the logarithm of the total number of configurations gives the entropy per vortex, which therefore is reduced by a constant $\sim k_{\mathrm{B}} \ln 2$. This leads to a free energy cost $-T \Delta S \sim\left(k_{\mathrm{B}} T\right)^{2} L /\left(\tilde{\epsilon} \ell^{2}\right)$ for a single Josephson vortex. Including this with the energetic contributions from the first and last terms in Eq.2.11 gives the free energy density (per unit area $L W$ )

$$
f \sim-r \frac{\epsilon_{J}}{\ell \lambda_{J}}+\frac{\epsilon_{J}}{\lambda_{J}^{2}} e^{-\ell / \lambda_{J}}+\frac{\left(k_{\mathrm{B}} T\right)^{2}}{\epsilon_{J}} \frac{\lambda_{J}}{\ell^{3}},
$$

where $r=\left(H / H_{c 1 J}-1\right)$. For $k_{\mathrm{B}} T \ll \epsilon_{J}$, the minimum free energy is determined by the balance between the first and second terms, leading to

$$
\ell_{T=0} \sim \lambda_{J} \ln (1 / r)
$$

or, in terms of the internal average magnetic field, $\langle B\rangle \sim$ $\phi_{0} /\left(\lambda_{J} d \ln (1 / r)\right)$. For sufficiently small $r$, or at high temperatures, however, this law breaks down, due to the effects of the entropic term. The true asymptotic behavior is obtained by balancing the first and last terms to give

$$
\ell_{\text {asymp. }} \sim \frac{k_{\mathrm{B}} T}{\epsilon_{J}} \frac{\lambda_{J}}{\sqrt{r}} .
$$

The crossover to this behavior is, however, extremely close to the CIT at typical experimental temperatures.
It occurs when $r<r_{c o} \sim\left(k_{\mathrm{B}} T / \epsilon_{J}\right)^{2} / \ln ^{3}\left(\epsilon_{J} / k_{\mathrm{B}} T\right)$, which for, say, a niobium junction with $d \approx 1500 \AA$ at $T=4.2 \mathrm{~K}$, requires $r \lesssim 10^{-8}$. These thermal effects might, however, be more observable near the bulk superconductornormal transition (especially in high temperature superconductors) where the Josephson coupling energy vanishes (within mean field theory) like $\sqrt{1-T / T_{c}}$.

\section{JUNCTIONS WITH DEFECTS}

The junctions of Ref. 10 contain defects, which are regions in which the local thickness is substantially increased. For columnar defects parallel to the $y$ axis, Eq.2.1 must be modified to $j_{0} \rightarrow \alpha(x) j_{0}$, with $\alpha(x) \leq 1$. We have assumed that the patterning is uniform in the $y$ direction (columnar defects), so that $\alpha$ is independent of $y$. The tunneling current has a strong (exponential) dependence when the local thickness variation $\delta d \gtrsim \xi$, the superconducting coherence length. The expressions for the magnetic field (Eq.2.2) also become position dependent through the factor of $1 / d(x)$, but we will ignore this weaker dependence in what follows. 18

The modulation in $j_{0}(x)$ results in a modified sineGordon equation,

$$
\lambda_{J}^{2} \nabla^{2} \gamma=\alpha(x) \sin \gamma .
$$

The boundary conditions, Eq.2.4, are unchanged.

The modified sine-Gordon equation leads naturally to defect-related corrections to the dilute fluxon free energy, Eq.2.11. To compute them, we first write the modified sine-Gordon free energy corresponding to Eq.3.1.

$$
F=\epsilon_{\mathrm{J}} \int d x d y\left\{\frac{1}{2}|\nabla \gamma-\mathbf{h}|^{2}+\lambda_{J}^{-2} \alpha(x)(1-\cos \gamma)\right\} .
$$

Inserting $\gamma(x, y)=\sum_{i} \gamma_{0}\left(x_{i}(y)\right)$, keeping only the leading (one-body) term and ignoring an additive constant, gives a correction to Eq2.11 of

$$
\Delta \mathcal{F}_{N}=\tilde{\epsilon} \int d y \sum_{n} U\left(x_{n}(y)\right)
$$

where

$$
U(x) \equiv \frac{1}{4} \int \frac{d x^{\prime}}{\lambda_{J}} \frac{\alpha\left(x+x^{\prime}\right)}{\cosh ^{2}\left(x^{\prime} / \lambda_{J}\right)} .
$$

It has been pointed out by several authors 8 that an appropriate spatially dependent $\alpha(x)$ can lead to an increase in the critical current of a JJ. In section IV] we will demonstrate this explicitly for the case of periodic defects. In the remainder of this section, howerer, we will demonstrate that, contrary to several claims 8.9 , the critical current density of a large $\left(W, L \gg \lambda_{J}\right) \mathrm{JJ}$ with columnar defects is always zero in the thermodynamic 
limit 19 The critical current density also vanishes for the long $\left(W \gg \lambda_{J}, L \ll \lambda_{J}\right)$ "in-line asymmetrical" geometry considered in Ref. 8.20 The vanishing of the critical current density results from the screening of current inherent in the sine-Gordon equation. Even in zero applied magnetic field in a pure junction, the critical current density decreases with the system size once it is larger than $\lambda_{J}$.

To prove that the critical current density indeed vanishes, we will take a more concrete model of the disorder. Specifically, $\alpha(x)$ is taken to be piecewise constant on intervals of variable length. On all intervals, we require $0<\alpha<1$, the upper bound corresponding to a perfect junction (in fact, it is only necessary that $\alpha$ is bounded above and below by arbitrary constants). The lengths of the intervals should also be well behaved, so that the mean length $\sum_{i} x_{i} / N=\bar{x}$ exists. For simplicity, we consider the one-dimensional junction (i.e. $L \ll \lambda_{J}$ ) studied in Ref. 8.

Consider the mean current density for the junction,

$$
\bar{j} \equiv \frac{1}{W} \int_{0}^{W} d x j_{0} \alpha(x) \sin \gamma,
$$

where $\gamma$ is the solution of the one-dimensional sineGordon equation,

$$
\lambda_{J}^{2} \frac{d^{2} \gamma}{d x^{2}}=\alpha(x) \sin \gamma
$$

Multiplying by $j_{0}$ and integrating over $x$ gives

$$
\bar{j}=j_{0} \lambda_{J}^{2}(v(W)-v(0)) / W,
$$

where $v(x)=d \gamma(x) / d x$. Multiplying Eq.3.6 instead by $\lambda_{J}^{-2} v(x)$ and integrating from $x$ to $x^{\prime}$, one finds

$$
E\left(x^{\prime}\right)-E(x)=\lambda_{J}^{-2} \sum_{x<x_{i}<x^{\prime}} \delta \alpha_{i} \cos \gamma\left(x_{i}\right),
$$

where $x_{i}$ is the coordinate of the boundary between the $i^{t h}$ and $(i+1)^{t h}$ constant region, and $\delta \alpha_{i}$ is the jump in $\alpha$ at that boundary. The function $E(x)=v(x)^{2} / 2+$ $\alpha(x) \cos \gamma(x)$. Using $\left|\delta \alpha_{i}\right| \leq 1$ and the existence of $\bar{x}$, the magnitude of the right hand side of Eq.3.8 is bounded above by $\lambda_{J}^{-2} N=\lambda_{J}^{-2}\left|x-x^{\prime}\right| / \bar{x}$ (for large $L$ ). Since the $\cos \gamma$ term in $E(x)$ is order one, we have

$$
\left|v\left(x^{\prime}\right)^{2}-v(x)^{2}\right| \leq C \lambda_{J}^{-2}\left|x-x^{\prime}\right| / \bar{x}
$$

Here and in the remainder of this section, $C$ indicates any dimensionless constant of order one. Consider first the case where $v(W)$ and $v(0)$ have the same sign. Then $\left|v(W)^{2}-v(0)^{2}\right|=|(v(W)-v(0))(v(W)+v(0))|>$ $|v(W)-v(0)|^{2}$. Using Eq.3.7, this gives the bound $|\bar{j}| \leq C j_{0} \lambda_{J} / \sqrt{\bar{x} W}$. If $v(W)$ and $v(0)$ have opposite signs, then there exists some $x^{*}$ between 0 and $W$ with $v\left(x^{*}\right)=0$. Both $x^{*}<W$ and $W-x^{*}<W$, so $|v(W)-v(0)|=|v(W)|+|v(0)| \leq 2 C \lambda_{J}^{-2} W / \bar{x}$, which leads to a similar bound. We have therefore established in general that

$$
|\bar{j}| \leq C j_{0} \frac{\lambda_{J}}{\sqrt{\bar{x} W}}
$$

which vanishes in the thermodynamic $(W \rightarrow \infty)$ limit.

This result can be understood in terms of an amusing analogy. Consider a simple pendulum in a time varying gravitational field. If the gravitational constant cannot become negative or exceed it's normal value, Eq.3.6 describes this situation with $\gamma$ giving the angle of the pendulum and $x$ taking the role of time. By Eq.3.7, a nonzero critical current density would be equivalent to saying that one could continuously accelerate the pendulum by varying the gravitational field. Clearly, as the pendulum begins to move faster, the field must be switched from strong to weak more and more rapidly to continue to give it an average acceleration. This would require a vanishing $\bar{x}$, corresponding to the infinitesimal time interval in the large $W$ limit.

\section{PERIODIC DEFECTS}

Although a finite critical current density is impossible for an infinite system, an increased critical current can be obtained in finite systems by introducing pinning. A natural choice for such pinning is to choose a defect periodicity commensurate with the Josephson vortex spacing in the junction. Such junctions have been studied extensively in Refs. 10,11, and we discuss a theoretical approach to these junctions here.

For ease of presentation, we will assume that the defect configuration is such that the unit cell can be chosen even. The extension to unit cells without symmetry is straightforward. In this case, the phase is governed by Eq.3.1, where $\alpha(x)$ can be written in the form

$$
\alpha(x)=\sum_{m=0}^{\infty} \alpha_{m} \cos m q x,
$$

where $q=2 \pi / a$, with defect lattice spacing $a$. Making the change of varjables $\gamma=n q x+\eta$ and inserting Eq.4.1. Eq.3.1 becomes2

$$
\begin{aligned}
\lambda_{J}^{2} \nabla^{2} \eta= & \sum_{m=0}^{\infty} \frac{\alpha_{m}}{2}\{\sin [(n+m) q x+\eta] \\
& +\sin [(n-m) q x+\eta]\} .
\end{aligned}
$$

Under this transformation, the form of the boundary conditions (Eq.2.4) remains unchanged (with $\eta$ replacing $\gamma$ ), except that the $y$ component of the magnetic field is replaced by an effective value

$$
\tilde{H}_{y}=H_{y}-\frac{n q \phi_{0}}{2 \pi d}
$$


To simplify further, we will assume that the defect lattice is not too large, so $a \lesssim \lambda_{J}$, and that the field has been chosen close to commensurate, so that $\tilde{H}_{y} d \lambda_{J} \ll \phi_{0}$. Under these assumptions, $\eta$ will be slowly varying, and aلl the sines but the second $m=n$ term will average out 22 (unless $n=0$, in which case both $m=0$ terms are equal). Eq. 4.2 then reduces to a uniform sine-Gordon equation,

$$
\tilde{\lambda}_{J}^{2} \nabla^{2} \eta=\sin \eta
$$

where the effective Josephson penetration depth is

$$
\tilde{\lambda}_{J}=\left\{\begin{array}{ll}
\sqrt{2 /\left|\alpha_{n}\right|} \lambda_{J} & n \neq 0 \\
\sqrt{1 / \alpha_{0}} \lambda_{J} & n=0
\end{array} .\right.
$$

Although we have not discussed time dependence in any detail, exactly the same mapping holds if it is included through the usual $\lambda_{J}^{2}\left(\bar{c} \partial_{t}^{2}+\beta \partial_{t}\right) \gamma$ terms on the left hand side of Eq.2.3. Thus the statics and dynamics near the commensurate fields will behave as in a uniform junction, with an $n$-dependent effective penetration depth and shifted magnetic field. For small $\tilde{H}_{y}$, the physics will therefore extremely closely mimic that of the uniform case. In particular, the critical current at the $n^{t h}$ peak is simply evaluated at zero field. Thus

$$
I_{c n}=f\left(L / \tilde{\lambda}_{J}\right) L W \tilde{j}_{0}=f\left(L / \tilde{\lambda}_{J}\right) L W j_{0} \frac{\lambda_{J}^{2}}{\tilde{\lambda}_{J}^{2}},
$$

where $f(\chi) \rightarrow 1$ for $\chi \lesssim 1$ and $f(\chi) \sim 2 / \chi$ for $\chi \gg 1$.

As an example, we explicitly compute the effective parameters for the case studied experimentally in Ref. 10. There, the thickness of the defects is large enough $(d \gg \xi)$ to render $\alpha=0$ on the pinning sites. In this case, the function $\alpha(x)$ is a periodic train of rectangular pulses of width $a-w_{d}$ and height 1 , where $w_{d}$ is the defect width, separated by spaces of width $w_{d}$ with $\alpha=0$. Choosing the origin at the center of a defect, the Fourier series in Eq.4.1 can be inverted to yield

$$
\alpha_{n}=\left\{\begin{array}{ll}
-2 \sin \left(\pi n w_{d} / a\right) /(\pi n), & n>0 \\
1-w_{d} / a, & n=0
\end{array} .\right.
$$

This implies an effective Josephson penetration depth of

$$
\tilde{\lambda}_{J}=\left\{\begin{array}{ll}
\lambda_{J} \sqrt{\pi n / \sin \left(\pi n w_{d} / a\right)}, & n>0 \\
\lambda_{J} / \sqrt{1-w_{d} / a}, & n=0
\end{array} .\right.
$$

For an effectively long or small junction (note that this only requires $\tilde{\lambda}_{J} \gtrsim L$, not $\left.\lambda_{J} \gtrsim L\right)$, this gives the critical current $I_{c}=L W j_{0}\left|\sin \left(\pi n w_{d} / a\right) /(\pi n)\right|$ for $n>0$ and $I_{c}=L W j_{0}\left(1-w_{d} / a\right)$ for $n=0$. In this limit, these results have been obtained in Ref. 10 using a simple current blocking model. We point out here that the region of validity of these results is actually much wider than would be naively predicted, because the increased effective Josephson penetration depth brings the system further into the small junction limit. The predicted modification of the commensurate critical current due to the increased effective screening length (obtained from Eqs.4.6 and 4.8) has recently received strong experimental support.11

Further, we can discuss in this way the width of the commensurate peaks. For $\tilde{\lambda}_{J} \ll W$, in the overlap geometry considered here, the width of a peak is determined by the screening condition $\tilde{H}_{y} \sim \tilde{H}_{c 1 J} \sim \phi_{0} / d \tilde{\lambda}_{J}$. Using Eq.4.8, this gives

$$
\Delta H_{y, n}^{\text {screened }} \sim\left\{\begin{array}{ll}
\frac{\phi_{0}}{d \lambda_{J}} \sqrt{\sin \left(\pi n w_{d} / a\right) / n \pi}, & n>0 \\
\frac{\phi_{0}}{d \lambda_{J}} \sqrt{1-w_{d} / a}, & n=0
\end{array} .\right.
$$

We note in passing that an in-line geometry, in which the current is injected transverse to the magnetic field, has considerably different behavior in this limit, due to the restriction of the current to screening layers near $x=$ $0, W$. In the unscreened limit (still the overlap geometry) where $\tilde{\lambda}_{J} \gg W$,

$$
\Delta H_{y, n}^{\text {unscreened }} \sim \frac{\phi_{0}}{d W} .
$$

Between the two scaling regimes there is presumably a smooth crossover (which could, in principle, be calculated from a solution of the uniform sine-Gordon equation).

\section{TILT RESPONSE OF A SINGLE FLUX LINE}

As discussed in section II, the fluxon density vanishes continuously as $H$ is reduced toward $H_{c 1 J}$. Once the mean spacing $\ell \gtrsim 10 \lambda_{J}$, say, the exponentially decaying interactions between Josephson vortices are weak, and it is reasonable to attempt to approximate the solitons as independent. In fact, at finite temperature in an infinitely long $(L=\infty)$ system, thermally excited transverse wandering of the vortices actually invalidates this approximation. In normal experimental conditions away from the bulk superconductor-normal transition, however, $k_{\mathrm{B}} T / \epsilon_{J}$ is so small (c.f. the paragraph after Eq.2.14) that this fluctuation effect is negligible for typical sample lengths. A more serious problem is the extremely weak divergence of $\ell$ near $H_{c 1 J}$ given in Eq2.13 means that it is necessary to reach a reduced magnetic field of $r \lesssim e^{-10} \approx 5 \times 10^{-5}$ to get to this regime.

It is nevertheless interesting from a theoretical point of view to consider the limit of an isolated flux line. Such a situation is somewhat more realizable in three dimensions in a bulk superconductor, a case into which we may hope to gain insight through this simpler model. It also represents a soluble limit, even in the case of a random distribution of defects. We discuss this interesting random case next at $T=0$.

For simplicity, we define the slope of the applied magnetic field $h \equiv \frac{H_{x}}{H_{y}}$. Combining Eq.2.11 and Eq.3.3, the free energy for a single flux line is given by 


$$
\begin{aligned}
\mathcal{F}= & \int_{0}^{L} d y\left[\frac{1}{2}\left|\frac{d x(y)}{d y}-h\right|^{2}+U(x(y))\right] \\
= & -h(x(L)-x(0))+\int_{0}^{L} d y\left[\frac{1}{2}\left|\frac{d x(y)}{d y}\right|^{2}\right. \\
& +U(x(y))]+ \text { constant }
\end{aligned}
$$

where we have set the overall energy scale $\tilde{\epsilon}=1$ for simplicity. Since we are neglecting thermal effects, the physics is completely independent of this choice.

This free energy is also the expression for the classical action of a particle of mass 1 in $1+1$ dimensions in a potential $-U[x]$ where $y$ corresponds to the time coordinate for the classical particle. Note that this mapping is somewhat different from the usual boson analogy. 12 Since $-h(x(L)-x(0))$ is a boundary term, it does not effect the equations of motion for the classical particle. The quantity

$$
\tilde{E}=\frac{1}{2}\left|\frac{d x}{d y}\right|^{2}-U(x(y)) .
$$

corresponds to the energy of the classical particle system and is thus conserved. This means that the local slope of the flux line $\frac{d x}{d y}$ is a function only of the local potential $U[x]$ and of a single constant of the motion $\tilde{E}$.

The density of states for the flux line potential $U$ is

$$
D(u)=\lim _{W \rightarrow \infty} \frac{1}{W} \int_{-W / 2}^{W / 2} d x \delta(u-U[x])
$$

For now, we will require that the potential $U$ is bounded from below, which means there exists a

$$
u_{\text {min }}=\min U>-\infty .
$$

Note that $D(u)=0$ for $u<u_{\min }$.

We will compute the tilt response, defined as the slope

$$
\theta(h) \equiv \frac{x(L ; h)-x(0 ; h)}{L}
$$

of the flux line in its ground state. For small angles, $\theta \approx\left\langle B_{x}\right\rangle /\left\langle B_{y}\right\rangle$. If $h=0$, the minimum energy solution is $x(y) \equiv x_{0}$ where $U\left[x_{0}\right]=u_{m i n}$, i.e. the flux line in pinned to the deepest point of the random potential. This yields a free energy of

$$
\mathcal{F}=u_{\min } L
$$

If the magnetic field is applied at a nonzero slope $h$, it is possible that the flux line remains pinned on the deepest point, yielding the exact same value for the Hamiltonian. However, it is also possible that the flux line becomes unpinned and traverses the system at some average slope $\theta$ defined as above. Using Eq.5.3, the magnitude of the local slope is given by

$$
\left|\frac{d x}{d y}\right|=\sqrt{2[\tilde{E}+U(x(y))]} .
$$

It is a simple exercise to show that, if the upper endpoint $x(L)$ is free and the lower endpoint is at an absolute minimum of $U(x)$, the minimal path with always have $d x / d y \geq 0$ for $h>0$. It should be noted that, for an unpinned path $(d x / d y>0), \tilde{E}>-u_{\min }$, so that the argument of the square root is nonnegative. In the language of the classical particle this is just the requirement that the total energy of the particle is larger than the potential of the highest hill, so it has enough energy to traverse the system. Integrating Eq.5.8, the inverse slope $\alpha \equiv \frac{1}{\theta}=\frac{L}{x(L)-x(0)}$ is

$$
\alpha(\tilde{E})=\frac{1}{x(L)-x(0)} \int_{x(0)}^{x(L)} d x \frac{d y}{d x} .
$$

In the limit of a large system when the slope $\theta$ is nonzero, the flux line samples all of the disorder in the potential equally. Thus since the probability that a given point $x$ has potential $U[x]=u$ is just the density of states $D(u)$, Eqs.5.8 and 5.9 give

$$
\alpha(\tilde{E})=\int d u \frac{D(u)}{\sqrt{2(\tilde{E}+u)}},
$$

so long as $\theta \neq 0$. This relation implicitly determines $\tilde{E}$ as a function $\alpha$. The slope is determined by optimizing over all possible values of $\tilde{E}$. It should be noted that Eq 5.10 is completely independent of any spatial correlations in the function $U(x)$ so long as the system is large enough that the flux line samples all of the disorder equally.

By using Eq.5.3 to eliminate $U(x)$, Eq.5.2 can be rewritten, up to an additive constant, as

$$
\mathcal{F}=-h(x(L)-x(0))-\tilde{E} L+\int_{x(0)}^{x(L)} d x \frac{d x}{d y},
$$

Taking $L \rightarrow \infty$,

$$
\begin{aligned}
\mathcal{F}(\tilde{E}, h) & =L\left[-\frac{h}{\alpha(\tilde{E})}-\tilde{E}\right. \\
& \left.+\frac{1}{\alpha(\tilde{E})} \int d u D(u) \sqrt{2(\tilde{E}+u)}\right] .
\end{aligned}
$$

Extremizing $\mathcal{F}$ with respect to $\tilde{E}$ gives

$$
h=\int d u D(u) \sqrt{2(\tilde{E}+u)}
$$

which in principle, can be inverted to yield $\tilde{E}(h)$ and the tilt response $\theta(h)$ (cf. Eq.5.10). For future use, we note in passing that

$$
\frac{d h}{d \tilde{E}}=\alpha(\tilde{E})
$$


For some values of $h$, there will be no $\tilde{E}$ satisfying Eq.5.13. In this case, there is no finite optimal value of the inverse slope $\alpha$, and the ground state of the flux line is pinned with $x(y)=x_{0}$, and $U\left[x_{0}\right]=u_{\text {min }}$ so that $\theta=1 / \alpha=0$. As $h$ is increased from zero, the slope $\theta$ remains zero (pinned) until $h$ reaches some critical value $h_{c}$, where $\theta$ begins to increase (unpinned) to nonzero values. We therefore have $\partial B_{x} /\left.\partial H_{x}\right|_{h=0}=0$, i.e. there is a transverse Meissner effect.

The behavior near $h_{c}$ constitutes a critical phenomenon analogous to that at the lower critical field. Using the optimal value of $\tilde{E}$ found in Eq.5.13 in Eq.5.12, the optimal free energy for a given $h$ is

$$
\min \mathcal{F}(\tilde{E}, h)=-\tilde{E}(h) L
$$

as $L \rightarrow \infty$. This free energy for the flux line is equal to the energy of a pinned flux line (Eq.5.7) when $\tilde{E}$ obtains its minimal value $-u_{\min }$. In terms of the language of the classical particle, this simply says that the classical particle becomes "unpinned" as soon as it has sufficient energy $\tilde{E}$ to get over the highest hills of the potential. We will be able to expand the necessary equations around the critical value $\tilde{E}=-u_{\min }$ and more closely examine the transitional behavior.

\section{An Explicit Example}

To illustrate these results, consider a disorder potential with the uniform density of states given by

$$
D(u)=\left\{\begin{array}{lll}
-1 / u_{\min } & \text { for } & u_{\min }<u<0 \\
0 & & \text { otherwise }
\end{array}\right.
$$

with $u_{\text {min }}<0$. Eq.5.10 can be explicitly integrated to yield

$$
\alpha(\tilde{E})=\frac{\sqrt{2}}{-u_{\min }}\left[\sqrt{\tilde{E}}-\sqrt{\tilde{E}+u_{\min }}\right] .
$$

As discussed above, the critical value of $\tilde{E}$ is given by $\tilde{E}_{c}=-u_{\min }$, so the critical value of the slope of the flux line is

$$
\theta_{c}=\frac{1}{\alpha_{c}}=\frac{1}{\alpha\left(\tilde{E}_{c}\right)}=\sqrt{\frac{-u_{\min }}{2}} .
$$

Similarly, Eq.5.13 can be explicitly integrated to give

$$
h(\tilde{E})=\frac{2 \sqrt{2}}{-3 u_{\min }}\left[\tilde{E}^{3 / 2}-\left(\tilde{E}+u_{\text {min }}\right)^{3 / 2}\right]
$$

which yields a critical slope of the applied field

$$
h_{c}=h\left(\tilde{E}_{c}\right)=\frac{2}{3} \sqrt{-2 u_{\min }} .
$$

For slopes of the applied field that are less than $h_{c}$, the flux line is pinned at $\theta=0$. When $h$ reaches $h_{c}, \theta$ jumps to $\theta_{c}$, and then increases continuously as $h$ is further increased. In this particular case, Eq.5.17 can also be explicitly inverted to yield

$$
\tilde{E}(\alpha)=\frac{1}{4}\left[\frac{\sqrt{2}}{\alpha}-\frac{\alpha u_{m i n}}{\sqrt{2}}\right]^{2},
$$

By substituting Eq.5.21 into Eq.5.19, the complete (albeit messy) analytic form for the function $h(\alpha)$ can be found. In general, however, $\alpha(\tilde{E})$ is not explicitly invertible, so an analytic form for $h(\alpha)$ can not be obtained. Furthermore, the complete function $h(\alpha)$ is clearly very sensitive to the precise form of the density of states. Thus, we would like to focus on physics that is in some sense more universal.

\section{Angular Exponent Near The Depinning Transition}

To this end we consider the behavior near the critical slope. For $h-h_{c}$ small but positive, we should have the relation

$$
\left(h-h_{c}\right) \sim\left(\theta-\theta_{c}\right)^{\gamma}
$$

for some exponent $\gamma$. As long as $\theta_{c}$ is nonzero, this then implies

$$
\left(h-h_{c}\right) \sim\left(\alpha-\alpha_{c}\right)^{\gamma} .
$$

In general, the value of the exponent $\gamma$ will only depend on behavior of the density of states $D(u)$ very near to $u_{\text {min }}$. In the above example, using the analytic form for $h(\alpha)$, it is trivial to expand around $\alpha_{c}$ to get $\gamma=2$. In fact, $\gamma$ will take the same value for all systems where the density of states $D(u)$ has a finite jump discontinuity at $u_{\min }$.

Let us suppose that the density of states takes the form

$$
D\left(u_{\min }\right) \sim\left(u-u_{\min }\right)^{\beta}
$$

for $u$ close to but greater than $u_{\text {min }}$ for some exponent $\beta$. Of course we must have $\beta>-1$ such that the integral of $D$ is finite and $D$ is normalizable.

We are now concerned with the behavior for $\tilde{E}$ close to but greater than $-u_{m i n}$. We define

$$
\Delta \tilde{E}=\tilde{E}+u_{m i n}
$$

With $\Delta \tilde{E}$ small, Eq.5.10 implies (with $p=u-u_{m i n}$ )

$$
\alpha(\tilde{E}) \sim \int_{0}^{q} d p p^{\beta}(p+\Delta \tilde{E})^{-1 / 2}+\cdots
$$

where $q$ is some small cutoff beyond which $D(u)$ no longer has the scaling form 5.24. This integral is finite for $\beta>$ $-\frac{1}{2}$, and otherwise diverges as

$$
\alpha(\tilde{E}) \sim(\Delta \tilde{E})^{\left(\beta+\frac{1}{2}\right)} \quad \text { for } \beta<-\frac{1}{2} .
$$


On the other hand, Eq.5.13 implies that $h(\Delta E)$ is always finite. But from Eq.5.14, we have $d h / d \Delta \tilde{E} \sim \alpha$ finite as $\Delta \tilde{E} \rightarrow 0$ only for $\beta>-\frac{1}{2}$ as described above. Finally,

$$
\frac{d \alpha}{d \tilde{E}} \sim \int_{0}^{q} d p p^{\beta}(p+\Delta \tilde{E})^{-3 / 2}+\cdots
$$

which is finite for $\beta>\frac{1}{2}$ and diverges as

$$
\frac{d \alpha}{d \tilde{E}} \sim(\Delta \tilde{E})^{\left(\beta-\frac{1}{2}\right)} \quad \text { for } \beta<\frac{1}{2} .
$$

In the case of $\beta>\frac{1}{2}$, we have $h, \alpha=d h / d \tilde{E}$, and $d \alpha / d \tilde{E}$ all finite and nonzero as $\Delta \tilde{E} \rightarrow 0$. Thus, expanding around $\Delta \tilde{E}=0$, we have $\left(h-h_{c}\right) \sim\left(\alpha-\alpha_{c}\right) \sim$ $\left(\theta-\theta_{c}\right) \sim \Delta \tilde{E}$, so $\gamma=1$, with $h_{c}>0$ and $\theta_{c}=1 / \alpha_{c}>0$.

In the case of $\frac{1}{2}>\beta>-\frac{1}{2}, d h / d \tilde{E}=\alpha$ is again finite as $\Delta \tilde{E} \rightarrow 0$, so $\left(h-h_{c}\right) \sim \Delta \tilde{E}$, but $d \alpha / d \tilde{E}$ diverges as $\Delta \tilde{E}^{(\beta-1 / 2)}$, so $\left(\alpha-\alpha_{c}\right) \sim \Delta \tilde{E}^{(\beta+1 / 2)}$. Thus $\gamma=1 /\left(\beta+\frac{1}{2}\right)$ with $h_{c}>0$ and $\theta_{c}>0$. Here we recover the result $\gamma=2$ for $\beta=0$ that we derived in the explicit example above.

In the case of $-1<\beta<-\frac{1}{2}, \alpha=d h / d \tilde{E}$ is divergent as $\Delta \tilde{E}^{(\beta+1 / 2)}$ as $\Delta \tilde{E} \rightarrow 0$, so $\left(h-h_{c}\right) \sim \Delta \tilde{E}^{(\beta+3 / 2)}$. Here $\alpha_{c}$ is divergent, so $\theta_{c}=0$. Thus, $\gamma=-\frac{\beta+3 / 2}{\beta+1 / 2}$, and Eq.5.23 does not hold.

For $\beta$ approaching $-\frac{1}{2}$, the exponent $\gamma$ diverges. For $\beta=-\frac{1}{2}$, Eq. 5.28 can be integrated for small $\Delta \tilde{E}$ to yield $\alpha \sim-\ln (\Delta \tilde{E})+\cdots$ which is divergent for small $\Delta \tilde{E}$ so $\theta_{c}=0$. Since $d h / d \tilde{E}=\alpha$, we have $\left(h-h_{c}\right) \sim$ $\Delta \tilde{E}(\ln \Delta \tilde{E}-1)$. Thus,

$$
\left(h-h_{c}\right) \sim e^{-1 / \theta}\left(\frac{1}{\theta}-1\right) \quad \text { for } \quad \beta=-\frac{1}{2}
$$

\section{Finite Sized Systems}

In the case of a finite sized system, a flux line with nonzero slope can only sample all of the disorder equally if $|x(L)-x(0)|$ is much larger than the correlation length of the disorder. If $|x(L)-x(0)|$ is not much larger than the correlation length, we can not use the above method to average over the disorder, and we must treat the correlations explicitly.

For example, in any finite sized system with smooth disorder (ie, $U(x)$ has nonzero correlation length), there is some genericly unique point $x_{0}$ such that $U\left(x_{0}\right)=$ $u_{\text {min }}$. Near this point, we can expand $U(x)$ as

$$
U(x)=u_{\min }+A\left(x-x_{0}\right)^{2}+\ldots
$$

For $h=0$, the lowest energy solution is the flux line pinned at $x_{0}$. For nonzero $h$, the flux line should pull away from this minimum in a continuous manner. In the simple case of $U(x)=A x^{2}$, (choosing $u_{\min }=0$, and $x_{0}=0$ ), we can study this behavior explicitly. For simplicity we fix one end of the flux line to be pinned in the middle of this deepest well, that is, $x(0)=x_{0}=0$, and we allow $x(L)$ to vary. Then $L, \tilde{E}$, and $x(L)$ are all related by the restriction

$$
L=\int_{x(0)}^{x(L)} d x \frac{d y}{d x}=\int_{x(0)}^{x(L)} d x \frac{1}{\sqrt{2(\tilde{E}+U(x))}}
$$

which can be integrated explicitly in this parabolic case and solved to yield

$$
\tilde{E}=A[x(L)]^{2}[\sinh (L \sqrt{2 A})]^{-2} .
$$

Now differentiating Eq.5.11 with respect to $x(L)$ (and again using Eq.5.8 to find a minimum of the free energy, yields the general result (compare to Eq.5.13)

$$
h=\sqrt{2[\tilde{E}+U(x(L))]},
$$

which in the case of the parabolic potential yields

$$
x(L)=h[\sqrt{2 A} \operatorname{coth}(L \sqrt{2 A})] .
$$

This calculation can equally well be performed in the case where we allow both endpoints to vary and the result is similar. Of course this result holds only in the range where $x(L)$ is small enough such that the potential $U$ still looks parabolic. Furthermore, if $x(L)$ becomes sufficiently large that there is a point $x_{1}$ far away such that $U\left(x_{1}\right)<U(x(L))$ then it is possible that the flux line will stretch to a more favorable position near $x_{1}$. Nonetheless, for a generic finite sized system, for $h$ sufficiently small, the slope $\theta=[x(L)-x(0)] / L$ will vary as $\theta \sim h$. However, as can be seen from Eq.5.35, as $L$ gets larger, the range of $h$ values for which this parabolic approximation is valid is reduced exponentially. For larger values of $h$ or for larger $L$, the system may then cross over to behavior more like the infinite sized system discussed above.

\section{CONCLUSION}

In this paper, we have discussed two types of commensurability effects in Josephson junctions containing columnar defects. The first kind is tuned by changing the magnitude of the applied magnetic field along the direction of the defects. For periodic arrangements of the pins, the vortex lattice induced by an applied magnetic field locks in to reciprocal lattice vectors which are integral multiples of the defect array wavevector (fractionally commensurate states also occur, but are suppressed by several powers of $a / \lambda_{J}$, as discussed in appendix A). Near these states, the critical current of a typical junction is substantially enhanced, though the critical current density remains zero in the thermodynamic limit. 
A second type of commensurability can be investigated by varying the transverse component of the field, or its direction. This effect is present for many distributions of the defects, though the character of the transition to the incommensurate (unaligned) state depends upon this distribution. In the case of periodic defects, the mapping of section IV shows that a commensurate vortex lattice remains locked in until a finite threshold transverse field is reached, after which kinked fluxons enable the array to tilt. The transverse constitutive relation $B_{\perp}\left(H_{\perp}\right)$ was found, under rather general conditions, to be simply a rescaled version of the zero parallel field result. We also investigated the dilute limit (near $H_{c 1 J}$ ) where each vortex acts independently. Using a mapping to one-dimensional particle mechanics, we showed that in most random distributions of pins, the fluxon makes a first order jump to a non-zero tipping angle as the transverse field is increased to a critical value. Above this field, this angle increases continuously, and near the critical field this increase can be described by an almostuniversal scaling exponent. Similar discussions of this "tilt response" have been given for the case of a finite density of fluxons with randomly placed defects in Ref. 23. In a periodic array of defects away from commensuration, and in the single vortex limit, the effect does not exist at finite temperatures, as discussed in Ref. 24.

Recent experimental work on long overlap junctions has confirmed many of our theoretigal conclusions for the case of the periodic defect array.10.11 In particular, the critical currents at the commensurate peaks agree quite well with the estimate following Eq4.8, with small deviations which are still well understood within the model. Although the peak widths are less reliably obtained, they appear to be consistent with Eqs.4.9 and 4.10.

A particularly dramatic consequence was reported in Ref. 11. There, the I-V characteristics were measured above $I_{c}$ at various commensurate magnetic fields. These $\mathrm{I}-\mathrm{V}$ curves showed "commensurate field steps," analogous to the zero field steps seen at zero field. Upon rescaling the current axis by $\left[\tilde{\lambda}_{J}(n=1) / \tilde{\lambda}_{J}(n=0)\right]^{2}$, the steps for $n=0$ and $n=1$ collapsed to a single curve. This dynamic measurement confirms the generality of the mapping. Another potential characterization is the number of zero field steps (which is roughly $L / 2 \tilde{\lambda}_{J}$ ), but an accurate determination requires an impractically large system width. It would be interesting to further explore experimentally and theoretically the consequences of this mapping.

Another interesting possibility from the theoretical point of view is a "floating" phase, in which a commensurate fluxon array is prevented from locking into the periodic defect array by thermal fluctuations. As discussed in II, the large value of $\epsilon_{J} /\left(k_{\mathrm{B}} T\right)$ renders thermal fluctuations unimportant unless $1-T / T_{c} \ll 1$. Such a floating phase may, however, be possible near the bulk superconducting transition, along with other interesting fluctuation effects not discussed here. Experimental observation of such a state would no doubt be difficult due precisely to these noisy fluctuations. We must leave these experimental and theoretical questions open.

\section{ACKNOWLEDGMENTS}

It is a pleasure to acknowledge helpful conversations with Mark Itzler, Michael Tinkham, and Onuttom Narayan. We would also like to thank Mark Itzler for making his experimental data available to us prior to publication. This research was supported by the National Science Foundation under Grant No. PHY89-04035 at the ITP, and National Science Foundation Grant DMR91-15491 at Harvard.

\section{APPENDIX A: HALF-INTEGER COMMENSURATE STATES}

In this appendix, we extend the treatment of section IV to handle the behavior near the half-integer commensurate fields. This condition is defined (at the center of a peak) by

$$
H_{y}^{(k / 2)}=\frac{k}{2} \frac{q \phi_{0}}{2 \pi d},
$$

where $k$ is an odd integer (for $k$ even this condition reduces to that for the integral peaks discussed earlier). Higher denominator fractional commensurate states should also occur, but the treatment becomes considerably more complicated and will not be described here.

Proceeding as before, the change of variables $\gamma=p x+$ $\eta$, with $p=k q / 2$, leads to the equation

$$
\begin{aligned}
\lambda_{J}^{2} \nabla^{2} \eta= & \sum_{m=0}^{\infty} \frac{\alpha_{m}}{2}\{\sin [(p+m q) x+\eta] \\
& +\sin [(p-m q) x+\eta]\}
\end{aligned}
$$

Unlike Eq.4.2, Eq.A2 does not contain any nonoscillatory terms. More precisely, a first order perturbative expansion in $\alpha$ will give convergent results. Nevertheless, we expect that the fluxon array should lock in to the defect lattice for small $\tilde{H}_{y}$. In fact, the halfinteger commensurate effect shows up as a divergepce in the second order perturbative solution of Eq.A2.25 To understand the nature of this divergence, we make the (non-linear) change of variables from $\eta$ to $\sigma$, where

$$
\eta=\eta_{0}(\sigma, x)+\sigma,
$$

where

$$
\begin{aligned}
\eta_{0}= & -\sum_{m} \frac{\alpha_{m}}{2 \lambda_{J}^{2}}\left[\frac{\sin [(p+m q) x+\sigma]}{(p+m q)^{2}}\right. \\
& \left.+\frac{\sin [(p-m q) x+\sigma]}{(p-m q)^{2}}\right] .
\end{aligned}
$$


To motivate Eq.A3, note that $\eta_{0}$ is the lowest order solution which would have been obtained from Eq.A2, taking a constant value of $\eta=\sigma$ on the right hand side. Despite the non-linearity,

$$
\begin{aligned}
\nabla^{2} \eta \approx & \nabla^{2} \sigma+\sum_{m} \frac{\alpha_{m}}{2}[\sin [(p+m q) x+\sigma] \\
& +\sin [(p-m q) x+\sigma]]
\end{aligned}
$$

up to oscillatory terms which may be neglected to lowest non-trivial order in $1 /\left(\lambda_{J} p\right)$ for small $\tilde{H}_{y}$. At this level of approximation, Eq.A2 becomes

$$
\begin{aligned}
\lambda_{J}^{2} \nabla^{2} \sigma & =\sum_{m} \frac{\alpha_{m}}{2}\left[\sin \left[(p+m q) x+\eta_{0}+\sigma\right]\right. \\
& +\sin \left[(p-m q) x+\eta_{0}+\sigma\right]-\sin [(p+m q) x+\sigma] \\
& -\sin [(p-m q) x+\sigma]] .
\end{aligned}
$$

The leading (linear) contribution in a Taylor series in $\eta_{0}$ gives the result

$$
\begin{aligned}
\lambda_{J}^{2} \nabla^{2} \sigma= & \sum_{m=0}^{\infty} \frac{\alpha_{m}}{2} \eta_{0}[\cos [(p+m q) x+\sigma] \\
& +\cos [(p-m q) x+\sigma]] .
\end{aligned}
$$

By inserting Eq.A4 into Eq.A7, combining sines and cosines, and neglecting oscillatory terms independent of $\sigma$, one finds

$$
\begin{aligned}
\lambda_{J}^{2} \nabla^{2} \sigma= & \sum_{m, m^{\prime}} \frac{\alpha_{m} \alpha_{m^{\prime}}}{8 \lambda_{J}^{2}}\left\{\frac{1}{\left(p-m^{\prime} q\right)^{2}}\left[\sin \left(\left[\left(m+m^{\prime}\right) q-2 p\right] x-2 \sigma\right)-\sin \left(\left[\left(m-m^{\prime}\right) q+2 p\right] x+2 \sigma\right)\right]\right. \\
& \left.-\frac{1}{\left(p+m^{\prime} q\right)^{2}}\left[\sin \left(\left[\left(m+m^{\prime}\right) q+2 p\right] x+2 \sigma\right)-\sin \left(\left[\left(m-m^{\prime}\right) q-2 p\right]-2 \sigma\right)\right]\right\} .
\end{aligned}
$$

Keeping only the unmodulated contributions proportional to $\sin (2 \sigma)$, we arrive at the effective equation

$$
\tilde{\lambda}_{J}^{2} \nabla^{2} \tilde{\sigma}=\sin \tilde{\sigma}
$$

where $\tilde{\sigma} \equiv 2 \sigma$ and the effective Josephson penetration depth in this case is

$$
\begin{aligned}
\tilde{\lambda}_{J}= & \lambda_{J}\left\{\frac{\alpha_{k} \alpha_{0}}{4 \lambda_{J}^{2} p^{2}}-\frac{1}{4 \lambda_{J}^{2}} \sum_{m=0}^{\infty}\left[\frac{\alpha_{m} \alpha_{|m-k|}}{|p-(k-m) q|^{2}}\right.\right. \\
& \left.\left.+\frac{\alpha_{m} \alpha_{m+k}}{|p-(k+m) q|^{2}}\right]\right\}^{-1 / 2} .
\end{aligned}
$$

It should be noted that the change of variables (Eq.A3) from $\eta$ to $\sigma$ induces a complicated transformation of the boundary conditions in Eq.2.4. The corrections are, however, small by a factor of $1 /\left(\lambda_{J} p\right)^{2}$. Although we have not performed a detailed analysis of their effects upon the
JJ, we therefore expect only minor quantitative changes in behavior. Physically, one effect of these corrections is to account for overlap with the long "Fraunhofer" tails of the integer-field (including zero-field) commensurate peaks. A crude way of accounting for this effect is to treat the critical current, calculated as in Eq.4.6, as a shift relative to the (experimentally determined) Fraunhofer background.

Neglecting these complications, and taking into account the final change of variables from $\sigma$ to $\tilde{\sigma}$, the effective current and effective fields are

$$
\begin{aligned}
\tilde{H}_{y} & =2\left(H_{y}-H_{y}^{(k / 2)}\right), \\
\tilde{H}_{x} & =2 H_{x}, \\
\tilde{I} & =2 I
\end{aligned}
$$

where $H_{y}^{(k / 2)}$ is the commensurate field defined in Eq.A1.

From Eq.A10, it is clear that the half-integer states have critical currents suppressed by a factor of $\left(a / \lambda_{J}\right)^{2}$. We expect that higher denominator fractional states can also occur, but are suppressed by even higher powers of $a / \lambda_{J}$

We have estimated the magnitude of the critical current peak in the experiments of Ref. 10. Using Eqs.4.7. A10, 4.6, and A11, we obtain a lowest 1/2 integral peak reduced by a factor of approximately 600 from the zerofield critical current. Using $I_{c 0}=30 \mathrm{~mA}$ as determined experimentally, this gives $\Delta I_{c} \approx 0.05 \mathrm{~mA}$. A feature is barely visible in the critical current versus field plot (Fig.2 of Ref. 10), with a deviation from the background of roughly $0.2 \mathrm{~mA}$. Given the crude treatment of the boundary conditions and the smallness of the effect, such
${ }^{1}$ D. S. Fisher, M. P. A. Fisher, and D. A. Huse, Phys. Rev. B43, 130 (1991).

${ }^{2}$ D. R. Nelson and V. M. Vinokur, Phys. Rev. B48, 13060 (1993).

${ }^{3}$ R. H. Koch, V. Foglietti, W. J. Gallagher, G. Koren, A. Gupta, and M. P. A. Fisher, Phys. Rev. Lett. 63, 1511 (1989); P. L. Gammel, L. F. Schneemener, and D. J. Bishop, Phys. Rev. Lett. 66, 953 (1991).

${ }^{4}$ L. Civale, A. D. Marwick, T. K. Worthington, M. A. Kirk, J. R. Thompson, L. Krusin-Elbaum, Y. Sum, J. R. Clem and F. Holtzberg, Phys. Rev. Lett. 67, 648 (1991); M. Leghissa, L. A. Gurevich, M. Kraus, G. Saemann-Ischenko, and L. Ya. Vinnikov, Phys. Rev. B 48, 1341 (1993).

${ }^{5}$ Recent numerical work may yield better estimates of vortex and Bose glass critical exponents. See A. P. Young, J. Phys. A 26, L1067 (1993); H. S. Bokil and A. P. Young, unpublished.

${ }^{6}$ T. Natterman, I. Lyuksyutov, and M. Schwartz, Europhys. Lett. 16, 295 (1991); J. Toner, Phys. Rev. Lett. 67, 2537 
(1991); Y.-C. Tsai and Y. Shapir, Phys. Rev. Lett. 69, 1773 (1992).

${ }^{7}$ G. G. Batrouni and T. Hwa, Phys. Rev. Lett. 72, 4133 (1994).

${ }^{8}$ R. Fehrenbacher, V. B. Geshkenbein, and G. Blatter, Phys. Rev. B45, 5450 (1992).

${ }^{9}$ V. M. Vinokur and A. E. Koshelev, Zh. Eksp. Teor. Fiz. 97, 976 (1990) [Sov. Phys. JETP 70, 547 (1990)].

${ }^{10}$ M. A. Itzler and M. Tinkham, Phys. Rev. B., in press.

${ }^{11}$ M. A. Itzler and M. Tinkham, to appear in IEEE Trans. Appl. Supercon., (1994). See also M. A. Itzler, unpublished.

12 D. R. Nelson, Phys. Rev. Lett. 60, 1973 (1988); D. R. Nelson and H. S. Seung, Phys. Rev. B 39, 9153 (1989).

13 See, e.g. M. Tinkham, Introduction to Superconductivity, (McGraw-Hill, New York, 1975).

${ }^{14}$ C. N. Owen and D. J. Scalapino, Phys. Rev. 164, 538 (1967).

15 See, e.g. A. Barone, ed., Josephson Effect: Achievements and Trends (world Scientific Pub. Co., Singapore, 1986).

16 The asymptotic form (including prefactors) of $\tilde{\epsilon}$ and $V(\chi)$ is obtained by substituting $\gamma(x, y)=\sum_{i} \gamma_{0}\left(x-x_{i}(y)\right)$ in Eq.2.5, where $\gamma_{0}(x)$ is given in Eq.2.9.

17 See, e.g. S. N. Coppersmith, D. S. Fisher, B. I. Halperin, P. A. Lee, and W. F. Brinkman, Phys. Rev. B 25, 349 (1982).

18 The thickness dependence can be incorporated by including an additional term $-\lambda_{J}^{2} d^{-1} \nabla d \cdot \nabla \gamma$ on the left hand side of Eq.3.1.

19 This applies only to configurations of defects in which pinning sites do not become arbitrarily closely spaced as the junction size is increased. If this restriction is relaxed, as in the "optimized" junction of Ref. 8, a finite critical current density is indeed possible.

${ }^{20}$ For a long JJ of the overlap geometry considered in most of this paper, however, $\bar{j}$ need not vanish for $W \rightarrow \infty$. This is because the system is actually two dimensional, but not fully screened in the $y$ direction. If $L$ is taken to infinity as well, $\bar{j}$ will indeed vanish.

${ }^{21}$ This transformation is appropriate for studying the behavior near the "integral" commensurate states with $H_{y}=$ $\frac{n q \phi_{0}}{2 \pi d}$. "Fractional" states can also occur, and are discussed in appendix A.

${ }^{22}$ In fact, these terms give non-singular perturbative contributions, which are small in the limit considered. It is easy to show that the corrections are of order $\left(q \lambda_{J}\right)^{2}$.

${ }^{23}$ T. Hwa, D. R. Nelson, and V. M. Vinokur, Phys. Rev. B 48, 1167 (1993).

${ }^{24}$ L. Balents and D. R. Nelson, submitted to Phys. Rev. Lett., (1994).

${ }^{25}$ L. Balents and S. H. Simon, unpublished.

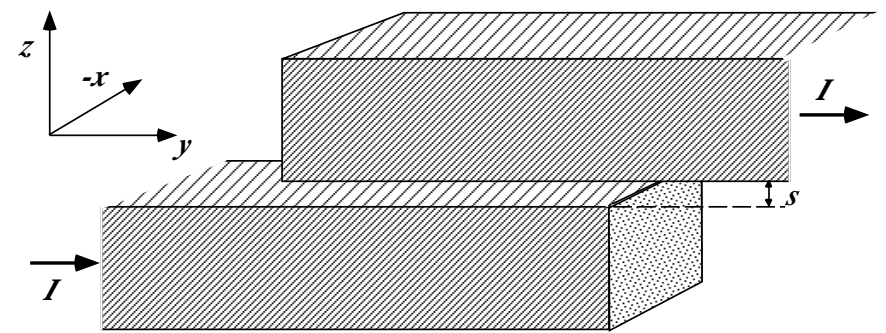

FIG 1: Overlap geometry for a large Josephson junction. 